\title{
Brasil y Colombia frente a la integración infraestructural: entre la construcción de región y el ostracismo*
}

\section{Brazil and Colombia Facing the Infrastructural Integration: Between Region Building and Ostracism}

\author{
Oscar Simmonds Pachón ${ }^{* * *}$
}

Recibido: 15/12/2013

Aprobado Evaluador Interno: 19/01/2014

Aprobado Evaluador Externo: 20/02/2014

\begin{abstract}
Resumen
Este escrito da cuenta de la posición de Brasil y Colombia frente a los procesos de integración infraestructural entendidos como parte de los procesos de regionalización en el espacio suramericano. Para ello, se parte del lugar que cada país ocupa dentro del sistema regional y la relación de esto con su despliegue dentro del sistema internacional. Luego, se analiza la manera en que ha sido abordada la integración infraestructural por parte de Colombia dentro de
\end{abstract}

\begin{abstract}
This paper reports on the position of Brazil and Colombia against infrastructure integration processes, understood as part of the process of regionalization in the South American territory. The study begins placing each of the countries within the regional system and the relationship of this with its deployment within the international system. After this, we analyze the way Colombia has addressed infrastructural integration inside its foreign policy agendas,
\end{abstract}

doi:10.11144/Javeriana.PAPO19-1.bcfi

* Artículo de investigación. El presente escrito se deriva y presenta apartes del proyecto de investigación Colombia frente a la Integración Infraestructural suramericana: una aproximación a su papel en la IIRSA, desarrollado en 2013 para optar al título de Magíster en Política Social de la Pontificia Universidad Javeriana. Dicha tesis fue dirigida por el Dr. Eduardo Pastrana Buelvas y obtuvo la distinción de Mención Honorífica.

** Administrador público de la Escuela Superior de Administración Pública (ESAP) y Magíster en Política Social de la Pontificia Universidad Javeriana. Profesor de tiempo completo del Programa de Relaciones Internacionales y Estudios Políticos de la Facultad de Estudios a Distancia de la Universidad Militar Nueva Granada y de la Maestría en Seguridad y Defensa Nacionales de la Escuela Superior de Guerra. Además, fue joven investigador del Instituto de Estudios Sociales y Culturales de la Pontificia Universidad Javeriana y (Pensar) y en la actualidad es investigador del grupo de investigción PIREO de la UMNG. Correo electrónico: oscar.simmonds@unimilitar.edu.co 
sus agendas de política exterior orientadas a las relaciones con sus vecinos, dando cuenta de la falta de una perspectiva estratégica por parte del Estado colombiano sobre el potencial liderazgo subregional que podría asumir en el marco de la integración infraestructural suramericana.

\section{Palabras clave:}

integración infraestructural; IIRSA; Potencia líder regional; potencia secundaria regional; política exterior; regionalismo oriented towards the relations with its neighbors, realizing the lack of a strategic perspective by the State on the potential subregional which could assume leadership within the South American infrastructure integration.

\section{Keywords:}

infrastructure integration; IIRSA; regional leader power; regional secondary power; foreign policy; regionalism 


\section{Introducción}

El papel de la Iniciativa para la Integración de la Infraestructura Regional Suramericana (IIRSA) en la integración infraestructural en Suramérica se constituye como una iniciativa intergubernamental entre los Estados de la región que apunta a generar las plataformas físicas necesarias para que estos países cuenten con los canales necesarios para avanzar en una interdependencia productiva y comercial que incremente los niveles de competitividad global de la región, y que se generen condiciones que permitan un mayor nivel de eficiencia en las cadenas productivas nacionales y regionales.

A partir de la disciplina de las Relaciones Internacionales, es claro que los procesos de integración infraestructural y los beneficios sociales y económicos que estos puedan generar dependen, en gran parte, de la posición que pueda tener cada Estado en la definición de agendas y temas de proyectos. En este sentido, en este artículo se parte de la posición de Brasil, en tanto potencia regional y principal jalonador de estas dinámicas a nivel sur-regional. En cuanto a Colombia, se entiende que este Estado ocupa el lugar de una de las potencias secundarias en la región, pues esta posición es compartida con Estados como el argentino, el chileno o el venezolano.

Tener presente lo anterior resulta fundamental a la hora de entender el rol que puede jugar cada país, el despliegue de su política exterior y las agendas tanto internas como externas en relación con un proceso como el de la integración infraestructural que se promueve desde la IIRSA. En términos prácticos, el lugar que cada uno de estos países ocupa termina por ser bastante determinante a la hora de definir asuntos como el nivel de generación de beneficios económicos y sociales, además de las responsabilidades relativas a los compromisos que pueden significar dicho proceso de integración para los países intervinientes.

A partir de esta primera aclaración, a continuación se plantea una estructura que da cuerpo a un escrito que aborda, inicialmente, algunos de los elementos de análisis que se derivan de la posición de Brasil y Colombia en el concierto regional e internacional. En la segunda sección se aborda el proceso de integración infraestructural adelantado por la IIRSA desde la perspectiva de la gobernanza multinivel, para luego entrar a dar cuenta de la manera cómo ha sido asumida por Colombia la integración física.

\section{Colombia y Brasil: sus posicionamientos regionales}

Brasil y Colombia ocupan posiciones distintas dentro de los sistemas regional e internacional, lo que supone amplias diferencias en las capacidades de despliegue exterior en estos dos sistemas. Mientras Brasil es la potencia regional indiscutible en Suramérica, Colombia ocupa el lugar de una de las potencias secundarias, es decir, que Colombia comparte su condición con otros Estados como Argentina, Venezuela y Chile, tradicionalmente (sin perder de vista la creciente relevancia en el escenario regional de países como Perú y Ecuador, sobre todo a partir de sus desarrollos en materia económica). 
Por tanto, a continuación se presentan algunos elementos de análisis que permitirán tipificar los distintos niveles de liderazgo que presentan estos países en la región y el grado de capacidad de agencia que efectivamente pueden ejercer en el sistema regional suramericano y en el sistema internacional.

\section{Brasil y su lugar como potencia regional emergente}

La política exterior brasileña de los últimos 15 años - finalización del gobierno de Cardozo, los dos gobiernos de Lula Da Silva y lo que lleva Dilma Rousseff- parece tener una característica fundamental y es que no se desliga de objetivos centrales de las políticas públicas estatales como la lucha contra la pobreza y el fortalecimiento del mercado interno en función de un mayor posicionamiento económico y político a nivel global. ${ }^{1}$ En razón de esto, los gobiernos brasileños de estos años han adelantado una serie de políticas sociales que van, hasta cierto punto, de la mano de las agendas de internacionalización y posicionamiento económico internacional.

Ejemplo de ello son dos '"políticas sociales" que han alcanzado renombre a nivel internacional: la "Bolsa familia" y la apuesta de estos gobiernos, sobre todo desde Lula, de garantizar un incremento sostenido del salario mínimo de millones de trabajadores brasileños (Pastrana y Vera, 2012). La "Bolsa familia" es una política que entrega un subsidio a los beneficiarios de entre 60 y 120 reales -entre 30 y 60 dólares americanos aproximadamente-, dependiendo de la cantidad de hijos que se tengan por familia. Esta política ha logrado, en cerca de diez años, alcanzar a más de 43 millones de brasileños y ayudar a salir de la pobreza extrema a más de 28 millones (Fleury, 2012).

En cuanto a la política de incrementar el salario mínimo, Brasil mostró cómo este pasó de estar en 200 reales en 2002 a llegar a los 510 reales en 2010 (Pastrana y Vera, 2012). Es decir, este ítem de la política laboral y económica brasileña mostró un crecimiento de más del $50 \%$ en ocho años, permitiendo que la capacidad de consumo

\footnotetext{
${ }^{1}$ En términos comparativos, hoy en día Brasil se ubica entre las principales economías del mundo y sus tendencias de crecimiento permiten pensar en que este país puede, en un futuro mediano, sobrepasar el PIB de varios de los países catalogados como del primer mundo. En este sentido, Fraga (2011) plantea que: "En los años setenta el problema de la región era que lo que la región producía perdía valor frente a los productos industriales del mundo desarrollado. En los años noventa el problema era que lo que la región producía perdía valor frente a la ciencia, la tecnología y el conocimiento que producía el mundo desarrollado. Y esto ha cambiado. Diez años atrás el PBI de América del Sur (todo reunido) era igual al PBI de Francia o Gran Bretaña, o California. Hoy el PBI de Brasil, que acaba de superar al de Italia, con lo cual Brasil ha pasado a ser la séptima economía mundial, queda inmediatamente después del PBI de Francia y el PBI de Gran Bretaña. Es decir, una década atrás Gran Bretaña o Francia equivalían a los 12 países de América del Sur reunidos en su PBI. Hoy prácticamente Brasil va a camino, en un año, dos años, a igualar el PBI de Francia o Gran Bretaña. Por dos razones, hemos crecido más que Europa y lo que producimos vale más que diez años atrás". (p. 96)
} 
de gran parte de la población mejorara. En este sentido, como anota Salama (2010), parte de la reducción de la concentración del ingreso en el gigante suramericano tiene que ver mucho con las políticas sobre cambios en los salarios de los trabajadores: "La disminución de las desigualdades en Brasil se explica principalmente por la mejora de las condiciones de trabajo (empleo, salario). Gracias, sobre todo, al aumento del salario mínimo y a la disminución relativa del empleo informal, la mejora de los ingresos de los asalariados y, fundamentalmente, de los ingresos bajos más que de los elevados” (p.13).

Ahora bien, más allá de los efectos sociales internos que medidas como las anteriores han generado, existe otro plano de análisis y de carácter internacional, en el que se puede ver la coherencia que existe entre estas políticas internas con la agenda externa de Brasil. Esta coherencia está dada en tanto que las políticas sociales de este país le sirven para proyectarse como "punta de lanza en la lucha internacional contra la miseria" (Pastrana y Vera, 2012) y al mismo tiempo convertirse en un una experiencia que puede servir de ejemplo a otros países de la región.

Por un lado, para un país que busca establecerse como potencia comercial resulta bastante conveniente contar con una sociedad con un buen nivel de consumo, lo cual le garantiza ciertos niveles de demanda y financiamiento, hasta el punto de darle el carácter de acreedor neto en la arena internacional, ${ }^{2}$ sobretodo en momentos en que las principales economías del mundo se encuentran en tiempos de desaceleración de sus tasas de crecimiento. Por otro lado, convertirse en un ejemplo en la lucha contra la pobreza puede significarle a Brasil avanzar en su posicionamiento como líder regional en asuntos como estos.

Es decir, esto le da la capacidad a Brasil de ejercer movimientos de poder blando al lograr que otros países traten de imitar sus ejercicios y vean en este país un aliado en sus luchas domésticas contra la pobreza. Para dar una muestra de lo anterior, y sin ir demasiado lejos, resulta bastante ejemplificante que el gobierno de otro de los líderes regionales a nivel latinoamericano, como es el caso de México, vea en Brasil un posible

${ }^{2}$ Como plantea Salama (2010), Brasil ha experimentado una variación en sus capacidades de financiamiento de tal manera que le han servido no sólo para mantener sus programas sociales, sino para adquirir una característica necesaria a la hora de situarse como potencia emergente y es la de ser acreedor neto. Así las cosas, el autor plantea lo siguiente: "Las capacidades de financiamiento continuaron aumentando fuertemente, y con ellas, las reservas: 1) las inversiones de portafolio (acciones, bonos y obligaciones) crecieron, pasando de 5,3 mil millones de dólares en 2003 a 9 mil millones en 2006, para caer fuertemente a -4,7 mil millones en 2009 durante la crisis, siendo las salidas superiores a las entradas;7 2) las inversiones extranjeras directas aumentaron considerablemente y se elevaron en un promedio de 19 mil millones de dólares por año entre 2000 y 2005. Alcanzaron los 45 mil millones de dólares en 2008 y, luego, con la crisis, cayeron a 26 mil millones, aunque deberían elevarse a 37 mil millones de dólares en 2010 de acuerdo con las previsiones. Las capacidades de financiamiento superaron las necesidades y por lo tanto, las reservas aumentaron considerablemente. Estas, ubicadas en el extranjero, permitieron que Brasil se transforme en un país acreedor neto" (p. 8). 
socio estratégico en la lucha contra sus problemas internos de pobreza. Respecto a esto, el portal de noticias de Telesur anota que el recién electo Presidente Mexicano, Enrique Peña Nieto, planteó la posibilidad de contar con el apoyo de Brasil en la lucha contra la pobreza mexicana que llega, según el mismo portal de noticias, a cerca del 50\% de la población: "El presidente electo de México sostuvo un encuentro este jueves con la presidenta de Brasil, Dilma Rousseff, en el que le pidió respaldo para emprender proyectos sociales en su nación que combatan la pobreza, ante el éxito que ha tenido el gigante de Suramérica en esta área” (Telesur, 2012).

Otro ejemplo en el que Brasil ha venido construyendo un liderazgo, esta vez con alcances más allá de Sur o Latinoamérica, es en cuanto el empuje a los procesos de democratización de instancias globales en materia económica y comercial. Muestra de ello es que buena parte de sus acciones en escenarios internacionales como la Organización Mundial del Comercio (OMC) tienden a presentar una imagen que apunta a catalogarlo como un líder regional que entiende la importancia de los problemas de países que han sido excluidos, tradicionalmente, de las instancias de toma de decisiones fundamentales, como son las de carácter comercial a nivel global:

Por tanto, Brasil se ha hecho consciente de la influencia que puede tener como potencia emergente, multiplicando su asociación estratégica con otras potencias semejantes, más allá de Suramérica como escenario de proyección regional. Al mismo tiempo, este país ha estado promoviendo la construcción de un orden más equitativo, con una mayor participación de los países del sur en la toma de decisiones. Como resultado de ello, se creó, por su iniciativa, en el marco de la OMC, el Grupo de los 20 (G-20 Comercial), esta es una alianza de países del sur que pretende defender sus intereses en la ronda de desarrollo de Doha. Asimismo, impulsó la constitución del foro de diálogo IBSA. En suma, Brasil no sólo ha diversificado sus intercambios comerciales, sino que ha venido desarrollando una política exterior universalista. (Pastrana y vera, 2012, p. 137)

A partir de estas consideraciones se puede ver una muestra del doble rol que ha adquirido Brasil, el de ser la principal potencia regional y el de ser, al mismo tiempo, una potencia mediana con pretensiones de potencia global. Es decir, dinámicas como la anterior le permiten a Brasil ser llamada, en palabras de Flemes (2012), Nolte (2006) y Pastrana (2011) una potencia regional emergente en ruta de posicionarse como uno de los actores internacionales más relevantes en las próximas décadas.

Ahora bien, esta prospección tiene un elemento explicativo muy importante y es que Brasil ha venido desarrollando un fortalecimiento institucional de su Estado que lo ha llevado a ser un agente fundamental en el desarrollo de la política brasileña de la última década. Siempre articulando esta gestión económica a la política exterior, de 
tal manera que el Estado se ha reestablecido como un agente negociador internacional de mucho peso y que trata de armonizar su avance como potencia con el bienestar de su sociedad y el mejoramiento de las condiciones socioeconómicas de sus vecinos regionales. Respecto a esto, Cervo (2010) enfatiza en lo que sería la nueva configuración de la política exterior brasileña en relación con sus objetivos de agenda socioeconómica interna, como se puede ver a continuación:

(...) nuevas facetas a la política exterior brasileña (...) dentro de las cuales se destacan (...): el reforzamiento del papel del Estado como un negociador internacional; la defensa soberana de los intereses nacionales, incluyendo grandes negocios en el proceso de globalizarse; las alianzas con países emergentes con objetivos idénticos, comenzando con los suramericanos; el diálogo abierto, en vez de la subordinación, con los países centrales; y un componente de moralidad en la lucha en contra de la pobreza y el hambre. (2010, p. 9)

Así las cosas, la creciente participación del Estado brasilero en procesos nacionales y regionales es uno de los aspectos claves que explica el creciente nivel de despliegue regional y mundial de Brasil. Ahora bien, esto no ha significado la vuelta a un Estado de carácter intervencionista o de planificación central en este país, sino que se ha tratado de una reconfiguración de la identidad misma del Estado brasileño, el cual ha tomado el rol de "negociador internacional” en un escenario de globalización económica, pero distanciándose en parte de las recetas tradicionales de las instancias multilaterales de crédito. ${ }^{3}$

Es decir, la experiencia brasileña no se ha fundamentado en las fórmulas que se derivaron del Consenso de Washington, las cuales apuntaban a una liberalización de los agentes del mercado en función de la aparente eficiencia que la plena libertad de estos agentes promovería.

Entonces, junto con los avances en ciertos indicadores asociados a las rentas de los diferentes segmentos de la población y la aparente sostenibilidad de programas como el de la "Bolsa familia", además de los incrementos en la productividad y en el comercio internacional, Brasil se encuentra en una situación que le exige garantizar su posición de punta de lanza en la región, con el fin de poder consolidar varios de

\footnotetext{
${ }^{3}$ Para Fleury (2012), la forma que el Estado brasilero se ha venido erigiendo como un agente fundamental en la economía nacional, de tal manera que esta sea un instrumento de avance de la unidad nacional, tiene asidero en una tradición de gobierno que se podría situar desde el gobierno de Getúlio Vargas. Para la autora: "La tradición brasileña de modernización conservadora o revolución pasiva viene de lejos, lo que para algunos autores como Vianna (2006) permite comprender el lulismo como el punto final del proyecto iniciado por Getúlio Vargas (en el poder entre 1930 y 1945), siempre pendiente de la construcción de una unión nacional que estuviera por encima de las clases, con el propósito de implantar un proyecto de desarrollo nacional" (p. 37).
} 
sus procesos en pro del desarrollo y del posicionamiento estratégico a nivel global que busca. En palabras de Pastrana y Vera (2012), Pinheiro da cuenta de estos aspectos que requieren la atención de Brasil:

1. Brasil es un país rico pero con grandes desigualdades internas en términos regionales, de ingreso y de acceso a la educación y la cultura. 2. Brasil experimenta tres grandes vulnerabilidades externas: militar, porque su aparato de defensa es aún insuficiente para el tamaño del territorio; de la población y del mar territorial; económica, por la dependencia relativa al ingreso de capital extranjero; y finalmente tecnológica, por el rezago visible en esta materia frente a las primeras economías del mundo. 3. Brasil tiene un gran potencial aún por explotar en términos de lo que puede producir su gran parque industrial, de lo que puede lograr su gran población con una mejor formación y de lo que puede hacer para acceder de manera sostenible a recursos naturales numerosos en un territorio que se conoce apenas en un $30 \%$. 4. Brasil aún tiene un "desafío democrático" o mucho por hacer para convertir la participación ciudadana en una fuente efectiva para la toma de decisiones a todo nivel, incluyendo la política exterior. (Pastrana y Vera, 2012, p. 150)

Entonces, además de los ajustes internos que significan estos retos, también es cierto que la región suramericana y la posibilidad de generar sinergias políticas y económicas con sus vecinos, juegan un papel muy importante en la proyección de Brasil y su política exterior, como también para la generación de las condiciones regionales que requiere para seguir promoviendo su desarrollo interno. A partir de los planteamientos de Pinheiro, Brasil necesitaría lograr el afianzamiento de la articulación y la interdependencia con los países de la región de tal manera que se formara una especie de comunidad de seguridad que no sólo le brindara estabilidad a sus políticas internas y externas, sino que al tiempo le permitiera, en tanto líder regional, orientar aspectos estratégicos del despliegue global de Suramérica en tanto región con una identidad común.

De cierta manera, para Brasil sería sumamente importante lograr direccionar la configuración de una identidad más común a todos los países de la región suramericana para que se pueda hablar de un "nosotros", como plantean Pastrana y Vera (2012) a propósito de los argumentos en este sentido de Goderhardt y Lembcke, y se generen las confianzas y las articulaciones necesarias para el desarrollo, por ejemplo, de cadenas productivas regionales que permitan hablar de una posición regional de Suramérica frente al sistema internacional y no de posturas aisladas.

Así las cosas, se puede entender que el avance en el liderazgo basado en poder blando y diálogo multilateral de Brasil, en tanto potencia regional emergente, puede suponer una reconfiguración de varios de los patrones de interacción regional en Suramérica, de tal manera que estos apunten de manera diferente y se orienten a la 
construcción de un cierto sujeto colectivo que se fundamente en una identidad construida de manera mancomunada.

Esto sin duda sería necesario para pensar en que la región suramericana se constituya como una comunidad de seguridad que no se limite a proveer de estabilidad a la potencia regional sino que permita el mejoramiento de las condiciones de todos los países en tanto agentes activos y no como subsidiarios de un líder.

Esto, sin embargo, es algo que no se puede hacer sólo en escenarios intergubernamentales o a nivel de política exterior, sino que es necesario incorporar las cadenas económicas y productivas de los países de la región, ante lo cual la integración infraestructural puede jugar un rol altamente estratégico como dinámica de gobernanza multinivel en la que, partiendo de los planteamientos de Hooghe y Marks (2004), no sea sólo el Estado nacional el que se encargue de configurar las redes regionales, sino que se puedan generar acoples regionales entre diferentes actores de distintas naturalezas

Ahora bien, desde un plano de análisis más cercano al realismo y hablando en términos concretos o materiales, para Brasil es bastante estratégico que los procesos que se adelanten desde instituciones de gobernanza regional como UNASUR, OTCA o IIRSA, para mencionar sólo unos ejemplos, logren ir más allá de las relaciones intergubernamentales, para que se logre contar con instituciones supranacionales que construyan agendas regionales más que nacionales. Desde una perspectiva de suma de poder se podría decir, como plantea Fraga (2011) que el interés de Brasil en UNASUR, para hablar de un caso, sería que un afianzamiento de este bloque comercial, bajo la orientación de Brasil, le permitiría incrementar su influencia sobre el uso y destinación, por ejemplo, de los recursos naturales que tienen estos países.

Mi impresión es que UNASUR hoy juega un papel importante en la política interior de Brasil. Es muy diferente si volvemos al mundo donde las potencias globales requieren cantidades: gran territorio, riqueza en recursos naturales y gran población. Cuando uno pone Brasil, que hoy es la séptima economía del mundo, en los pronósticos puede ser la cuarta. Y este es el punto. Si yo hago cantidades, y hablo de UNASUR, estas cantidades se duplican, se duplica el territorio, se duplica la población, se duplica el PBI. Y entonces América del Sur pasa a ser un actor global. (p. 104)

Sin duda, este análisis de sumas y restas también sirve para comprender en parte el rol de Brasil como potencia regional con pretensiones globales, pues la capacidad de contar con un mayor nivel de influencia sobre sus vecinos, a partir de su participación en instancias de gobernanza regional, como es el caso de la UNASUR, puede significar contar con mayores recursos a la hora de negociar con la otra cara de su posición, es decir, la de jugador global. 
Esto, entre otras cosas, implica que países como Colombia, que se mantienen en el renglón de las potencias regionales secundarias, tienen la necesidad de comprender muy bien los movimientos estratégicos de la potencia regional, sobre todo cuando se trata de temas como la integración infraestructural, la cual tiene importantes impactos en asuntos como las cadenas productivas trasnacionales (este tema será abordado con mayor profundidad más adelante). Es decir, como señala Herrera: "el ascenso de Brasil plantea interrogantes en cuanto a los desafíos y las oportunidades que trae para los otros Estados de la región" (2012, p. 566).

Además, tener claro que Brasil tiene un doble rol en tanto potencia, permitirá estar alerta a las dinámicas promovidas desde este mismo país y que pueden conllevar a que Colombia y otros países se limiten a ser beneficiarios o asistentes en los procesos de regionalización, dejando de lado la posibilidad de ser agentes reales en la definición de las agendas en los temas relacionados con el avance de los procesos de construcción de región en el espacio suramericano. Teniendo en cuenta esto, en la siguiente sección se aborda el rol de Colombia en tanto potencia secundaria y algunas de las implicaciones que esto puede tener para que el país se desenvuelva como un actor con capacidad de liderazgo en algunos temas de las agendas regionales.

\section{Colombia como potencia secundaria en la región suramericana: una posición condicionada}

En cuanto a la forma en que Colombia puede ser entendida en potencia secundaria a nivel regional, inicialmente hay que tener en cuenta una serie de atributos que, en términos generales, definen a una potencia secundaria. Para ello, resulta claro lo que plantean Pastrana y Vera (2012a) al respecto:

\footnotetext{
Las potencias secundarias son Estados que, en el contexto de una región determinada, poseen ciertos recursos de poder importantes, gracias a los cuales estarían en capacidad de retar o por lo menos frenar de forma gradual los objetivos de la potencia líder regional y competir de manera directa con otras potencias de capacidades semejantes (Flemes, 2010, p. 103). Las potencias secundarias constituyen una categoría especial de los posibles seguidores del líder regional, en este caso Brasil. Este, para velar por sus intereses en la región -o en parte del contexto global- depende de la cooperación o, por lo menos, de la tolerancia o consentimiento de los primeros. (p. 189)
}

Siguiendo esta lógica, las potencias secundarias en Suramérica, como Colombia, se caracterizan por estar por debajo de la potencia regional, en términos de capacidades e influencia en los procesos regionales. Sin embargo, estas potencias tienden a tener un cierto nivel de liderazgo, sobre todo en aspectos definidos y no se 
entienden a sí mismas como meros seguidores de las directrices que se establezcan desde la potencia líder.

En términos generales, las potencias secundarias no tienen la capacidad de condicionar a la potencia regional pero sí están en capacidad de coordinar o influir en las agendas externas e internas de otros países que tienen menos capacidades que ellas:

Las potencias secundarias pueden definirse como los Estados que ocupan la segunda posición más poderosa dentro de una jerarquía regional. La posición secundaria está determinada por sus relativas capacidades en términos materiales y/o de ideas (Cooper et al. 1991; Flemes/ Wojczewski 2011). Por eso, las potencias secundarias tienen una posición sistémica de dos caras: formulan sus estrategias de política exterior partiendo de la condición de inferioridad fáctica, percibida y/o anticipada en relación a la potencia principal, así como de superioridad respecto a los otros Estados de la región. (Flemes, 2012, p. 21).

No obstante, esta concepción se presenta desde una vertiente de análisis del realismo neoclásico y no agota todos los elementos que se deben tener en cuenta a la hora de pensar a Colombia como potencia secundaria. Sin duda, factores como el alto nivel de centralidad que tiene la agenda en materia de seguridad, en clave de estar asociada a las políticas que se formulan en esta materia desde los Estados Unidos, entre otros, son asuntos que condicionan de manera importante el rol de Colombia como potencia secundaria.

En este sentido, a continuación se presentan algunos elementos que pueden ser utilizados para entender cómo Colombia se despliega en el escenario regional, sobre todo en relación a los procesos de regionalización y su accionar en instancias de gobernanza multinivel.

Antes de dar cuenta del rol de Colombia como potencia secundaria en Suramérica y como primer elemento de análisis, no se puede dejar de lado que, sobre todo en las últimas décadas, Colombia se ha caracterizado por presentar una serie de factores que han condicionado sus agendas políticas tanto a nivel interno como externo. Para muchos analistas que pasan por enfoques teóricos que se mueven entre la Ciencia Política y las Relaciones Internacionales, es notorio que elementos como el bilateralismo, el presidencialismo y la securitización de las agendas públicas han hecho que el país tenga una tendencia muy marcada en materia de definición de políticas públicas en múltiples sectores (Pastrana, 2011; Solano y Dangond, 2010; Herrera, 2012).

Esta condición que ha presentado Colombia ha supuesto un cierto aislamiento con respecto a los procesos de regionalización que se vienen adelantando en la región, lo que de alguna manera ha hecho, salvo algunas excepciones, que Colombia participe como un agente con bajo nivel de liderazgo o influencia en la definición de las agendas multilaterales, por ejemplo, en escenarios de gobernanza multinivel como UNASUR, IIRSA y OTCA (Organización del Tratado de Cooperación Amazónica), entre otros. 
Sin embargo, por aspectos como su crecimiento económico, la población y el potencial en cuanto a explotación de recursos naturales, Colombia sigue siendo considerada como una de las potencias secundarias de la región (Flemes, 2012).

Siguiendo con este autor, en el caso de Suramérica existen varias potencias secundarias, aunque esto no implica que esta cantidad sea estática, pues en el caso de Suramérica no hay demasiadas diferencias entre otros países y las potencias que Flemes asume como secundarias. Basta con hablar del caso de Perú, país que ha venido presentando un importante repunte económico y que hoy en día cuenta con una gran diversificación de redes comerciales que van más allá de la región. Igualmente, además de la cercanía en cuanto a indicadores entre potencias secundarias, otra circunstancia que determina el escenario regional en el que se desarrollan las potencias secundarias es que en la región no se presentan tensiones que conlleven a los países a entrar en conflictos bélicos o agresiones físicas transfronterizas.

Esta situación permite, desde un punto de vista teórico, que las potencias secundarias no tengan que entrar en gastos asociados con una estrategia de seguridad orientada a enfrentar bélicamente a un enemigo regional. Esto, sin embargo, no quiere decir que no haya situaciones de tensión entre los Estados, lo que sucede es que estas tienden a ser mediadas en espacios multilaterales, y esto puede significar una ganancia para las instancias de gobernanza regional ya que sería normal que los países acudan a ellas para abordar sus diferencias. En este sentido, Flemes da cuenta de lo siguiente:

América del Sur es una zona de paz negativa en la que pueden existir tensiones pero en la que formas de impugnación de tenor coercitivo o militar no constituyen una opción viable (Kacowicz 1998), y en la que este tipo de enfoques conflictivos no forman parte del instrumentario estratégico de las potencias secundarias de cara a Brasil. Teniendo en cuenta las ganancias marginales que las potencias secundarias de América del Sur podrían esperar de la aplicación de estrategias coercitivas en sus relaciones con la potencia principal, las estrategias institucionales parecen ser el enfoque más prometedor para impactar la jerarquía regional de Estados. (2012, p. 20)

Desde este punto de vista y sin precisar mucho en el tema del posible despliegue de Colombia en IIRSA - tema que será abordado en más detalle en páginas siguientes- se podría pensar que Colombia, al no tener que incurrir en gastos de defensa contra sus vecinos, contaría con una situación que le permitiría asumir el rol de líder en ciertos aspectos de las relaciones regionales. Sin embargo, dadas las condiciones expresadas anteriormente, las estrategias que el país podría utilizar no estarían en el marco de la confrontación o de la competencia directa con la potencia líder regional, ya que las condiciones materiales e ideacionales de Colombia no presentarían la garantía suficiente para lograr imponerse a Brasil. 
Lo anterior resulta fundamental, pues entender la región como un espacio realmente estratégico significaría una variación importante en la perspectiva colombiana; más aún si se tiene en cuenta que Colombia, debido a elementos como la securitización de sus agendas y la bilateralización, ha sido entendido por sus vecinos como una especie de vector que se aleja de manera particular de las lógicas regionales o de los procesos de regionalismo adelantados en la región. Sin ir muy lejos, Pastrana (2011) da cuenta de cómo, por ejemplo, la fuerte tendencia a ver en Estados Unidos el país socio por excelencia ${ }^{4}$ han hecho que Colombia no cuente con una política exterior diversificada:

Aunque no sea recíproco, la intensificación de las relaciones comerciales con los EE.UU. y la renovación de la cooperación siempre han ocupado el primer lugar en la agenda diplomática colombiana. En la era Uribe, el Ministerio de Comercio contemplaba el TLC con los EE.UU. como "el camino para definir el trato comercial con el resto del mundo (p. 4).

Ahora bien, desde la perspectiva del realismo neoclásico (Flemes, 2012; Pastrana y Vera, 2012; Nolte, 2006), un país que se entiende a sí mismo como potencia secundaria en una región, más que acudir a una "fuerza” externa a la región, lo que debe es entender qué acoples estratégicos puede desarrollar con la potencia líder regional con el fin de que esta no se convierta en una hegemonía regional que haga de los demás países sus seguidores y subsidiarios.

Es decir, no se trataría de entrar en una pugna directa con el líder regional, como se planteó anteriormente, sino de lograr, en espacios de gobernanza regional, equilibrios de poder que permitan que los intereses de todos los países implicados sean definitivos a la hora de la construcción de agendas multilaterales y de políticas externas por parte de la potencia regional, incluso llegando a influir en sus políticas internas, si estás implican una alteración de las condiciones de los vecinos. De alguna manera esto supone el avance de la construcción de ese "nosotros" con una identidad regional más o menos construida de manera conjunta. Siguiendo con Flemes (2012), esta estrategia en la que las potencias secundarias buscan influir en la potencia regional, sin que esto

\footnotetext{
${ }^{4}$ En este sentido y como anotan Mauricio Solano y Claudia Dangond (2010), en el caso de Colombia las relaciones bilaterales con el país del norte han ocupado un lugar primordial: tan importante ha sido que año tras año Colombia enfila sus baterías diplomáticas y políticas buscando que el apoyo se renueve por parte del Gobierno de los Estados Unidos, para lo cual siempre se requiere la aprobación del Congreso de ese país.

Para el año 2009, debido a la crisis económica mundial, la ayuda se ha visto amenazada, razón por la cual el gobierno colombiano debió buscar otras estrategias para mantener los mecanismos de lucha contra el tráfico ilícito de narcóticos. Entre ellas se ubica el tan discutido acuerdo para renovar la presencia de militares estadounidenses en bases militares colombianas.
} 
signifique un relevo de liderazgo, se denomina soft balancing, término que en palabras de este autor puede ser entendido como:

(...) una opción estratégica para las relaciones de las potencias secundarias con la potencia regional en regiones con bajos niveles de conflicto, en la que la rivalidad es reemplazada por patrones competitivos. El propósito del soft balancing es equilibrar o mejorar la distribución asimétrica de poder existente, así como frustrar la realización de objetivos de política exterior del actor más poderoso de la región incrementando los costos de sus acciones (Pape 2005, Paul 2005). Mientras que la búsqueda consecuente y revisionista de un nuevo equilibrio de poder en el sentido de hard balancing involucra una reconfiguración del orden regional, como por ejemplo el fin del predominio de la potencia principal, el soft balancing apunta a obstaculizar y restringir el proceso de emergencia de tal forma que la potencia principal tenga en cuenta los reclamos y demandas de la potencia secundaria. Así, finalizar el predominio de una potencia ascendente no constituye el leitmotiv de la impugnación a través del soft balancing. (p. 20)

Además de los puntos anteriores hay que tener en cuenta un elemento que le da un tinte diferente al carácter de potencia secundaria de Colombia frente a las demás: Colombia es asumido, por cierta parte de los países de la región, como una posible fuente de desestabilización y de amenaza a la seguridad, debido fundamentalmente a la persistencia histórica del conflicto interno armado.

Sin embargo, sin ahondar demasiado en los detalles e implicaciones internacionales del conflicto interno, sí resulta importante dar cuenta de un efecto que ha tenido la forma como se ha enfrentado esta amenaza "doméstica" por parte de los últimos gobiernos colombianos. Tal factor es que Colombia, principalmente durante el gobierno del expresidente Uribe, asumió una postura frente a los países con los que comparte frontera el país que lo situaba como el único en la región con la capacidad para enfrentar la amenaza del conflicto, aún si esto significaba traspasar las fronteras. Como plantean Pastrana y Vera (2012b), Colombia se concibió como un agente con la capacidad y la necesidad de asegurar su seguridad interna, más allá de su territorio: acá resulta clave recordar el episodio del ataque al campamento de Raúl Reyes en 2008, por parte del ejército colombiano, lo que implicó bombardear territorio ecuatoriano.

Para finalizar esta sección y como se dijo anteriormente, sin ahondar en todas las implicaciones de esta postura, es importante mencionar que actitudes como estas le han significado a Colombia una serie de costos en términos regionales que se han manifestado sobre todo en la desconfianza que algunos países han expresado hacia Colombia y sus gobiernos. Esta situación, sin duda, constituye un peso muerto que de alguna manera limita el desenvolvimiento de Colombia en instancias de gobernanza regional y que 
necesita ser superada, si se quiere obtener un verdadero liderazgo subregional que vaya más allá de los foros multilaterales. Quizá esto implique que Colombia se muestre dispuesto a correr con algunos de los costos que implican los procesos de regionalización - este punto será abordado con mayor profundidad en capítulos posteriores-.

\section{IIRSA y la integración infraestructural en Suramérica: una aproximación desde la gobernanza multinivel}

La provisión de servicios de infraestructura a nivel regional puede ser entendida como uno de los aspectos fundamentales en los procesos tendientes a la formación de una integración regional sistémica (Cipoletta, 2011). Contar con la infraestructura regional que permita esta provisión resulta ser una condición sin la cual no se podría pensar en una integración regional que involucre los procesos productivos de los países y la generación de cadenas productivas regionales y subregionales que afiancen las interconexiones productivas y no sólo comerciales. ${ }^{5}$

Además, como como plantea Tavares (2013), esta estrategia se sustenta, desde la teoría económica, en las ventajas que ofrecen las economías de aglomeración ${ }^{6}$ para reducir costos de producción y mejorar los intercambios de mercancías y factores de producción. A propósito de esto, el autor propone lo siguiente:

En contraste con la fragilidad conceptual que había marcado a los proyectos de integración acordados entre estos países durante la segunda mitad del siglo pasado, la IIRSA fue formulada a partir de una fuente incuestionable de crecimiento de largo plazo, que es la

\footnotetext{
${ }^{5}$ Para la CEPAL y UNASUR, IIRSA: "Se trata de un mecanismo de cooperación y diálogo entre 12 países de América del Sur para intercambiar información y coordinar políticas y planes de inversión sectoriales. Tiene como objetivo promover la integración física en las áreas de transporte, comunicaciones y energía, procurando promover el desarrollo sostenible de la región. Su papel estratégico consiste en mitigar y, en lo posible, eliminar los obstáculos de integración física más notorios (cuellos de botella, tramos faltantes, etc.); fomentar el comercio intrarregional en América del Sur; estimular la reorganización de cadenas productivas; asistir en la construcción de una economía suramericana más integrada, competitiva y dinámica en un marco de sostenibilidad social y ambiental; incentivar la participación del sector privado; abogar por la armonización de políticas públicas y marcos regulatorios entre los países y sectores, y disminuir el costo comercial y de distribución mediante el desarrollo de infraestructura en los sectores de transporte, energía y telecomunicaciones" (2011, p. 52).

${ }^{6}$ El concepto de "economías de aglomeración" asume que la interconexión geográfica de diferentes procesos asociados a la producción puede generar reducciones en los costos de los agentes; "En definitiva, la tendencia de la actividad económica a concentrarse en puntos concretos, se explica por la existencia de economías de aglomeración, es decir, ventajas que obtienen las empresas BID por localizarse en un lugar determinado: la aglomeración da lugar a ganancias de productividad, que para la empresa se traducen en reducciones de costes y para la región en un desplazamiento hacia afuera" (Aláez, Longás y Ullibarri, 2001, p. 154).
} 
generación de economías de aglomeración, conforme se demuestra en algunos textos clásicos de la teoría económica, como Marshall (1920), Perroux (1955) y Krugman (1991). (p. 142)

Ahora, el montaje de infraestructuras productivas, de transportes, de comunicaciones y de flujos de energía (eléctrica, producida a base de hidrocarburos u otros minerales, entre otras) no sólo se debe entender en función de la generación de lazos regionales fuertes, sino que estas juegan un papel determinante en el desarrollo económico y social de las sociedades.

A partir de una relación positiva entre la infraestructura y el crecimiento económico, y de una relación negativa entre infraestructura y desigualdad de ingresos se deja en evidencia que aquellos países que tienen mejor disponibilidad de infraestructura son los que expresan mejores resultados de desarrollo económico (medido en términos de crecimiento interanual del PIB), al mismo tiempo que tienen menor inequidad en la distribución del ingreso. La validez de ambas relaciones se verifica tanto para la dotación física como para la calidad de la infraestructura provista; y su intensidad se magnifica especialmente cuando se involucra la infraestructura con la conectividad y las políticas de inclusión social apuntadas a las regiones que económica y socialmente se encuentran menos privilegiadas. (Cipoletta, 2011, p. 8)

Según esto, se puede pensar que el avance de una integración infraestructural a nivel regional en el que los diferentes países interactúen como agentes no determinados por alguno de los actores regionales, sino que su articulación se dé en un espacio de gobernanza multinivel, puede resultar en el incremento de la capacidad regional y de cada país en términos de su productividad. Teniendo en cuenta estos elementos de análisis, a continuación se mostrará uno de los esfuerzos de gobernanza regional más importantes en pro de la integración de la infraestructura del continente suramericano: IIRSA.

Lo anterior con el fin de aproximarnos de manera analítica a los avances y obstáculos que ha presentado esta Iniciativa, su relación con el avance del regionalismo en Suramérica, el rol que puede tener en el posicionamiento de la potencia regional y las potencias secundarias, tanto en función de su proyección regional y global como en la solución de ciertos problemas referentes al desarrollo social y económico de sus sociedades.

\section{IIRSA: principales características y despliegue regionales}

La IIRSA se constituye, quizá, en el principal esfuerzo de integración infraestructural en Suramérica. Esta iniciativa comenzó a ser gestada en una reunión que sostuvieron los mandatarios de los países de Suramérica en Brasilia entre el 31 de agosto y el 1 de septiembre del año 2000. En dicha reunión se planteó la necesidad de contar con una agenda coordinada en materia de modernización e integración de la infraestructura, 
esto en el entendido de ser pasos necesarios para promover de manera estructural el desarrollo social y económico de los países desde una perspectiva regional. En este sentido, dentro del Comunicado de Brasilia que se produjo como resultado de la reunión de mandatarios se asume, en el numeral 37 del documento, que:

Integración y desarrollo de la infraestructura física son dos líneas de acción que se complementan. La formación del espacio económico ampliado suramericano, que anhelan las sociedades de la región, dependerá de la complementación y expansión de proyectos existentes y de la identificación de otros nuevos proyectos de infraestructura de integración, orientados por principios de sostenibilidad social y ambiental, con capacidad de atracción de capitales extraregionales y de generación de efectos multiplicadores intraregionales. Avances en el campo de la infraestructura, por su parte, revertirán en nuevos impulsos para la integración, creándose así una dinámica que debe ser incentivada. Ese escenario sería también beneficiado por una política de inversiones con perspectiva regional y no sólo nacional. (p.7)

Luego, en diciembre del mismo año, los ministros de las carteras de transporte, energía y telecomunicaciones de Suramérica se reunieron en Montevideo para formular el Plan de Acción 2000-2010 que establecía las líneas de acción estratégica de la iniciativa. En dicho Plan se definieron nueve líneas de acción básicas que serán presentadas a continuación.

1. Diseñar una visión más integral de la infraestructura: esta línea busca que la integración infraestructural incorpore criterios de sostenibilidad ambiental y sinergia con las infraestructuras y equipamientos sociales de los territorios implicados.

2. Encuadrar los proyectos dentro de una planificación estratégica a partir de la identificación de los EIDs regionales: en esta línea se busca que los proyectos permitan el avance de las metas de los Ejes de Integración y Desarrollo definidos territorial y socialmente.

3. Modernizar y actualizar los sistemas regulatorios e institucionales nacionales que norman el uso de la infraestructura: buscar la compatibilidad de los marcos legales aparece en el Plan de Acción 2000-2010 como una condición para poder avanzar en la integración.

4. Armonizar las políticas, planes y marcos regulatorios e institucionales entre los Estados: esta línea apunta a que los procedimientos para el diseño de proyectos esté estandarizado para todos los países y así permitir la complementariedad de los diseños programáticos.

5. Valorizar la dimensión ambiental y social de los proyectos: los proyectos deben apuntar a una sostenibilidad ambiental a partir de marcos en la materia, construidos conjuntamente. 
6. Mejorar la calidad de vida y las oportunidades de las poblaciones locales en los ejes de integración regional: para la iniciativa resulta fundamental que las comunidades locales afectadas por los proyectos logren incrementar sus condiciones sociales y económicas.

7. Incorporar mecanismos de participación y consulta: tanto los agentes privados, como las comunidades locales, además de las instancias gubernamentales y multilaterales implicadas, deben contar con el espacio necesario para presentar sus opiniones sobre todos los proyectos de la iniciativa.

8. Desarrollar nuevos mecanismos regionales para la programación, ejecución y gestión de proyectos: poder consolidar la integración infraestructural requiere del diseño de mecanismos que superen las inflexiones de los marcos programáticos nacionales.

9. Estructurar esquemas financieros adaptados a la configuración específica de riesgos de cada proyecto: los proyectos deben prever la atención de los riesgos ambientales, sociales y culturales que puedan conllevar las ejecuciones de los proyectos.

A partir de estas líneas de acción se continuó, desde el año 2000, con una serie de reuniones entre ministros, jefes de Estado y representantes de estos que permitieron, en la Reunión de Cuzco de diciembre de 2004, la consolidación de la Agenda de Implementación Consensuada 2005-2010 (AIC) en la que se definieron 31 proyectos prioritarios y que tenían como plazo de ejecución la primera década de este siglo. En total, estos proyectos adquirieron un valor proyectado de US\$ 14 mil millones, los cuales deberían ser aportados por los países miembros de la iniciativa, principalmente (Tavares, 2013). Esta agenda incluyó como sus proyectos prioritarios, los siguientes:

21 proyectos referentes a puentes y rutas, tres pasos de frontera, dos trazados ferroviarios (anillo ferroviario de San Pablo y ruta Los Andes-Mendoza), dos proyectos en el área de comunicaciones (servicios postales y acuerdos de roaming entre operadoras de telefonía celular), un puerto fluvial en Ecuador, la recuperación de la navegabilidad por el río Meta -que discurre por territorios de Colombia y Venezuela-y la construcción de un gasoducto en el noroeste de Argentina. (Tavares, 2013, p. 143)

En términos de la sectorización geográfica que implementa IIRSA, es fundamental tener en cuenta que esta iniciativa desarrolla sus gestiones a partir de nueve ejes territoriales que son: eje del Amazonas, eje Andino, eje, de Capricornio, eje del Escudo de Guayanés, eje de la Hidrovía Paraguay-Panamá, eje Interoceánico central, eje Mercosur-Chile, Eje Perú-Brasil-Bolivia y eje del Sur. Estos Ejes de Integración y Desarrollo (EID) son a partir de los cuales se agrupan los proyectos y las inversiones establecidas para cada uno. 
En cuanto a los niveles de ejecución de los proyectos de la AIC, es claro que estos no han llegado a un cumplimiento pleno puesto que diferentes inconvenientes asociados principalmente a los problemas de incompatibilidad jurídica y de agendas estatales se han tornado bastante importantes y han limitado el avance de la iniciativa. Sin embargo, a pesar de estos y otros problemas se han presentado avances importantes, como lo plantea la UNASUR: "en suma, si se observa que el 61\% de los proyectos de la Agenda se encuentran en ejecución comparado con el 32\% que estaba en 2005 y 6,5\% ya está incluido, se pone de manifiesto que la agenda de integración de los países, si bien con distinto ritmo según el caso, se está concretando" (UNASUR, 2011, p. 109).

Otro momento importante en el avance de la planeación de la IIRSA se dio con el diseño de la Agenda de Proyectos Prioritarios de Integración (API). Esta agenda resultó como uno de los productos del Consejo Suramericano de Infraestructura y Planeamiento (COSIPLAN) - con un horizonte de ejecución entre 2012 y 2022-, instancia que se inscribe dentro de la UNASUR para el abordaje de los temas de infraestructura en la región. Este hecho, sin duda, permite pensar en un mayor nivel de articulación en términos de espacios de gobernanza multinivel y se puede pensar que el tema de la integración de la infraestructura regional, pues a pesar de ser un asunto sectorial, juega un rol fundamental en el avance del regionalismo en la región, valga la redundancia. Como elemento anexo a lo anterior, es importante tener en cuenta que durante los años que lleva la iniciativa, otras instancias de gobernanza regional, como el BID, se han vinculado a los proyectos de la iniciativa, sobre todo en términos de financiamiento:

Hasta diciembre de 2010, se habían financiado 28 proyectos de dicha cartera, valorados en US\$ 8.871 millones, con un aporte del Banco de US\$ 2.832 millones. En el periodo 2011-2012 se incrementa el aporte total del Banco en un 60\%, totalizando US\$ 4.552 millones, habiéndose aprobado un monto de US\$ 1.720 millones para cinco proyectos, lo que representa un incremento significativo con respecto al promedio anual de aprobaciones.

Esta tendencia deberá consolidarse a futuro. (BID, 2012, p. 34)

Entonces, en noviembre de 2011, durante la Segunda Reunión Ordinaria del COSIPLAN, celebrada en Brasilia, se formuló dicha agenda, la cual fue ratificada por los Presidentes de los países de la región durante la Sexta Reunión del Consejo de Jefas y Jefes de Estado de UNASUR que se realizó en el mes de noviembre de 2012 en Lima. Ahora bien, los proyectos de esta nueva agenda fueron propuestos teniendo en cuenta que estos debían ser concebidos como "proyectos estructurados" (IIRSA, 2011).

Es decir, que fueran capaces de consolidar redes de conectividad física a nivel regional, de tal manera que fueran potenciadores de las sinergias transfronterizas existentes y al mismo tiempo ser generadores de soluciones para las deficiencias y limitaciones de las 
infraestructuras existentes en los territorios donde se desarrollarán. Así las cosas, la API consta de 31 proyectos estructurados y que son entendidos como fundamentales para el avance de la regionalización integral que se promueve desde la UNASUR e IIRSA (Ver cuadro 1). Estos proyectos, que totalizan un monto de US $\$ 17.260 .7$ millones de dólares equivalen a un $13.3 \%$ del total de la Cartera del COSIPLAN al año 2012, que asciende a US\$130.139,1 millones (UNASUR, 2011, p. 5).

Fuente: IIRSA, 2012

\section{Cuadro No. 1}

Proyectos de la API por EID y monto de inversión (Millones de US\$)

\begin{tabular}{|l|c|c|c|}
\hline \multicolumn{1}{|c|}{ EID } & Proyectos estructurados & Proyectos individuales & Monto de inversión \\
\hline AMA & 3 & 25 & 3.418 .0 \\
\hline AND & 5 & 11 & 3.623 .9 \\
\hline CAP & 5 & 18 & 4.435 .4 \\
\hline GUY & 3 & 4 & 900.8 \\
\hline HPP & 4 & 15 & 1.998 .1 \\
\hline IOC & 4 & 7 & 416.7 \\
\hline MCC & 6 & 7 & 2.382 .3 \\
\hline PBB & 1 & 1 & 85.4 \\
\hline TOTAL & 31 & 88 & 17.260 .7 \\
\hline
\end{tabular}

Fuente: IIRSA, 2012

Asimismo, es necesario tener en cuenta que aunque los montos proyectados resultan ser importantes en cuanto a su tamaño, no todas las inversiones han llegado a ejecutarse ni en el tiempo ni en las cantidades establecidas en las agendas. ${ }^{7}$ Esto, sin embargo, no quiere decir que la iniciativa no haya avanzado de manera importante, pues más del 60\% de los proyectos de la API se encuentran en ejecución.

Entonces, en este paneo general sobre la gestión de la IIRSA, es conveniente dar cuenta de la forma en que ésta se ha desarrollado en el Eje Andino, al cual pertenece Colombia junto con Ecuador, Perú, Bolivia y Venezuela. En este sentido, se puede partir de que en este Eje están proyectadas inversiones superiores a los US\$ 3.600 millones que se distribuyen en 11 proyectos. De estos proyectos, el de mayor envergadura es el del

\footnotetext{
${ }^{7}$ Al respecto, Tavares (2013) es bastante crítico y plantea que los resultados rayan en lo decepcionante a la hora de hacer un balance de los resultados de la iniciativa en los últimos 10 años: "A pesar de las precauciones observadas en la selección de proyectos, los resultados de la AIC resultaron decepcionantes. En julio de 2010, el estado de la cartera era el siguiente: [I] dos proyectos concluidos, al valor de U\$S 22 millones (dos puentes); [II] 19 proyectos en ejecución, valorados en U\$S 7.700 millones; [III] 10 proyectos no iniciados, con valor estimado en U\$S 6.300 millones (IIRSA, 2011, p. 109)3. Sin embargo, de acuerdo con la visión del Comité de Coordinación Técnica de la IIRSA, estos resultados pueden considerarse como positivos" (pp. 145-146).
} 
corredor vial entre Caracas-Bogotá-Buenaventura-Quito con el fin de incrementar el transporte multimodal (integración de sistemas carreteros, férreos y fluviales), marítimo y carretero en la subregión.

A partir de estos datos, se puede ver que las agendas y los proyectos que se han venido formulando dentro del marco de la IIRSA, han apuntado, en su mayoría a esfuerzos tendientes a un mejoramiento de la interconectividad física de los países de la región. Sin duda, esto, como se ha planteado en páginas anteriores, aparece como una condición para superar el alto grado de intergubernamentalismo que ha permeado los procesos de regionalización en Suramérica.

\section{Una aproximación a la perspectiva colombiana de IIRSA}

Colombia ha sido un país que tradicionalmente ha privilegiado las relaciones internacionales con Estados Unidos (Pastrana y Vera, 2012; Dallanegra, 2012; Tickner, 2007). Esta postura se puede observar desde finales del siglo XIX y principios del XX, siendo a evidencia primigenia más relevante, la pérdida de Panamá.

Para Tickner (2007), esta forma de afrontar las relaciones internacionales se debe, en parte, a la idea que han tenido las élites colombianas según la cual la mejor manera de obtener buenos resultados en el sistema internacional es a partir de una asociación con los Estados Unidos, así esto signifique una pérdida de soberanía y de autonomía en la determinación de agendas políticas, tanto a nivel externo como interno (Pastrana y Vera, 2012a).

Aunque son múltiples las consecuencias de lo anterior, existe una que resulta bastante relacionada con los procesos de regionalización en Suramérica dentro de los cuales se encuentran los adelantados por IIRSA y es que Colombia se ha alejado de los procesos de construcción de región en el espacio suramericano. Es decir, Colombia ha dejado de lado los procesos que en Suramérica han tendido al fortalecimiento de lazos entre los países para mejorar su posicionamiento global como región.

Ejemplo de ello es que Colombia no ha demostrado un interés estratégico en los proyectos de la IIRSA, dejando de lado, incluso, el potencial que puede representar contar con uno de las instituciones pioneras en materia de interconexión eléctrica a nivel regional (ISA). Como anota Pastrana, refiriéndose al campo económico, el bajo nivel de sintonía con los procesos regionales

Ha sido un gran obstáculo a la aproximación regional la manera desequilibrada en la que se negoció con los norteamericanos, por encima de los arreglos comunitarios en la CAN, por fuera del proceso de integración comercial suramericano y haciendo caso omiso a las consecuencias regionales de no negociar en bloque, tal como lo han expresado países como Venezuela, Bolivia, Ecuador e incluso Brasil. Así, se desdibujó la profundización de las relaciones comerciales con el vecindario y el entorno regional e incluso se reversaron 
procesos de integración como el andino. Como consecuencia de ello, se fragmentó la CAN, Venezuela se orientó hacia el Mercosur y el gobierno de Hugo Chávez aceleró los procesos alternativos como el ALBA. (Pastrana, 2011, p. 4)

Entonces, en términos regionales lo que se puede ver es una posición condicionada por parte de Colombia debido a su sujeción a los Estados Unidos, lo cual además ha hecho que Colombia opte por desarrollar una estrategia de internacionalización económica y de desarrollo infraestructural interno de carácter individual en la cual no se logra la mejor articulación con los procesos que se podrían adelantar de manera conjunta con los países de la región, especialmente de los de la sub-región Andina. Este fenómeno ha estado matizado en varias oportunidades por tensiones entre Colombia y los países vecinos; siendo particularmente visibles los episodios de tensión con Venezuela y Ecuador (Márquez, 2012).

Situaciones como las anteriores se relacionan mucho con la elección de Colombia de apostarle a los Tratados de Libre Comercio como la base de la estrategia de la internacionalización, lo que conlleva a permitir la acción libre de los inversionistas extranjeros en Colombia. Esta situación termina por ser determinante a la hora de la definición de la forma cómo se invierte en la infraestructura nacional.

En este sentido, Colombia se ha convertido en uno de los principales destinos de la inversión extranjera de la región y buena parte de estas inversiones se destinan a la infraestructura, fundamentalmente en temas relacionados con el transporte y la capacidad exportadora del país. ${ }^{8}$ Como plantea la estrategia de la cuarta generación de concesiones, por parte de Colombia se busca generar las mejores condiciones de inversión para los agentes extranjeros, de tal manera que esto sean actores fundamentales en la promoción del desarrollo infraestructural del país. A propósito de esto, Tenjo presenta algunos datos de contexto que conviene tener en cuenta:

uno de los sectores que generan mayor número de proyectos es el de infraestructura, por esto se entiende que Colombia haya ocupado el "quinto lugar en inversión privada de infraestructura en la región de Latinoamérica y el Caribe en el periodo 1.990-2002” y actualmente que el total de inversiones previstas para infraestructura en el periodo 20062010 sean de 69 billones de pesos, donde alrededor de 20 billones de pesos corresponden al sector transporte, por ello la apropiación para el 2.009 es de 3.2 billones de pesos, es decir, Colombia ha invertido sostenidamente en infraestructura por encima del $2 \%$ del PIB, manteniendo el nivel de financiamiento público debido al incremento de la participación

\footnotetext{
${ }^{8}$ Como plantea Wills-Valderrama: "La construcción, la operación y el mantenimiento de infraestructura han sido reconocidos en Colombia como una de las locomotoras del desarrollo de la economía del país. Gran parte de la financiación para infraestructura proviene y provendrá del exterior; en su mayoría, en forma de inversión extranjera directa" (2011, p. 20).
} 
privada; claro está lo anterior atiende en primera instancia a los requerimientos del ALCA y en el presente a los tratados de libre comercio y las directrices IIRSA. (2009, p. 9)

Aunque esto puede significar un mayor incremento de la capacidad de la infraestructura nacional, también es cierto que buena parte de estas inversiones se destinan a la actividad exportadora de Colombia (transportes, fundamentalmente), de tal manera que supongan mayores niveles de eficiencia y menores precios en las actividades de transporte de mercancías hacia el exterior (principalmente Estados Unidos). Asimismo, esto se entrecruza con el modelo exportador de Colombia, el cual viene privilegiando los productos minero-energéticos como las principales mercancías (estas ocupan más del 60\% de la canasta exportadora del país), lo que puede resultar problemático porque estos no se caracterizan por llevar grandes niveles de valor agregado al momento de salir del país

Se puede pensar entonces, que, una parte de la falta de sintonía entre Colombia y la IIRSA, se debe a las diferencias de los modelos de desarrollo que están en juego: por un lado uno de carácter regional y enfocado a un mayor nivel de industrialización y eficiencia en el flujo de mercancías y mano de obra: el de IIRSA, y por otro lado, uno enfocado a la exportación de recursos naturales fuera del continente, como condición para una internacionalización económica, el de Colombia.

Teniendo en cuenta todo lo anterior, aparece entre muchas otras una situación problemática relevante: Colombia no cuenta con una postura frente a los procesos de integración infraestructural adelantados por IIRSA - entendidos como componentes de las dinámicas de regionalización en Suramérica- que le permita consolidarse como líder regional en la iniciativa y que evidencie un real compromiso del país con el avance de regionalización en el espacio suramericano.

Lo anterior es debido, entre otros factores, a la baja importancia que Colombia le ha dado a participar activamente en los procesos de regionalización y al privilegio que, tradicionalmente se le ha dado por parte de los gobiernos nacionales y muchas veces también por parte de los gremios, a los procesos de internacionalización a través de los Estados Unidos.

Así pues, el nivel de interacción, más allá de lo comercial, con los países y los vecinos regionales ha ocupado un lugar secundario en las agendas nacionales, lo que limita la capacidad del país para obtener mayores beneficios de estos procesos. En materia de infraestructura, estar fuera de la sintonía de los avances en la integración puede limitar la capacidad de la internacionalización económica que Colombia ha tratado de promover en las últimas décadas, ya que este alejamiento puede redundar en mayores costos de transporte, producción y montaje de cadenas productivas. Esta situación puede ralentizar el avance de la competitividad del país y el desarrollo que se podría generar con esta capacidad en el país, especialmente en los territorios que podrían interconectarse a través de la ejecución de los proyectos de la IIRSA. 
Para concluir esta sección, se puede decir que existen diversas restricciones por parte del Estado y el modelo de desarrollo colombiano que impiden que exista una verdadera articulación a los procesos de integración infraestructural. De alguna manera, esto puede significar que Colombia mantenga su tendencia extractiva, la cual le ha permitido situarse, en términos macroeconómicos, como un líder; claro está, sin que esto signifique que a nivel social las cosas estén mejorando al mismo ritmo. Para corroborar esto basta con recordar un simple indicador como el de la concentración del ingreso, dato que para el caso colombiano muestra que, según el Departamento Nacional de Planeación (DNP) (2012) se presenta un coeficiente de Gini de 0.539, lo que sitúa al país dentro de los diez países con mayores niveles de concentración del ingreso. Pensar en una regionalización que supere el nivel comercial y que permita al país contar con las plataformas infraestructurales enfocadas a los procesos productivos y no meramente a los extractivos resulta clave, pues la profundización del modelo aperturista de corte neoliberal puede seguir profundizando la debilidad de Colombia a la hora de producir mercancías con valor agregado, lo cual parece como necesario para lograr un verdadero desarrollo integral.

\section{Conclusiones}

En Suramérica, Brasil aparece como la potencia líder regional y existen países como Colombia, Chile, Argentina y Venezuela que pueden ser catalogados como potencias secundarias. Ahora, principalmente desde la potencia líder se han venido promoviendo, sobre todo desde las últimas dos décadas, esfuerzos que apuntan a la construcción de un regionalismo en la región que supere el nivel intergubernamental y aperturista que ha caracterizado la tendencia suramericana en la materia. Lo anterior puede ser interpretado como un despliegue estratégico de Brasil orientado a que América del Sur genere dinámicas que lo constituyan como un actor más o menos cohesionado, para lo cual es fundamental superar el nivel comercial que ha determinado la gran mayoría de las relaciones entre los Estados de la región.

$\mathrm{Al}$ aproximarse dentro de este marco al rol que países como Brasil y Colombia han tenido en materia de integración infraestructural, es clave ver cómo en IIRSA Brasil ha venido actuando como un agente determinante, mientras que Colombia ha presentado una baja participación y ha otorgado poca importancia a las agendas de los diferentes proyectos de la iniciativa.

Esta situación se puede explicar a partir del enfoque de desarrollo económico que Colombia ha venido ejecutando en las dos últimas décadas, sobre todo, y que apunta a una internacionalización de carácter neoliberal y que asume el sostenimiento y de pendencia de las relaciones con Estados Unidos como una condición para poder internacionalizar su economía, situación que se ha profundizado por la securitización de las agendas políticas a nivel interno y externo de Colombia. 
Esta postura de Colombia ha limitado el rol que el país podría tener en los procesos de integración regional y la posibilidad de actuar como un líder regional dentro de los procesos de regionalización en un tema tan estratégico a nivel global como el de la provisión de servicios de infraestructura. Así pues, si se quiere jugar un rol más determinante, el país tendría que disponerse a jugar un rol activo en materia de asumir costos de algunos de los componentes de la integración, iniciando desde un enfoque subregional, teniendo como espacio estratégico el Eje Andino de la IIRSA.

\section{Referencias Bibliográficas}

Aláez, R. Longás, C. y Ullibarri, M. (2001). La relación entre efectos externos y aglomeración: una aproximación a su estudio a partir de la evidencia empírica disponible. Revista de Estudios Regionales, 61, 151-167.

BID (2012). La integración de la infraestructura regional en los países andinos. Quito: BID.

Cipoletta, G. (2011). Principios de políticas de infraestructura, logística y movilidad basadas en la integralidad y la sostenibilidad. Santiago de Chile: CEPAL.

Cervo, A. (2010). Brazil's Rise on the Internacional Scene: Brazil and the World. Revista Brasileira de Política Internacional (Número especial), 23, 7-32.

Consejo Suramericano de Infraestructura y Planeamiento (2011). Agenda de Projetos Prioritários de Integração. Disponible en: www.iirsa.org.

Dallanegra, L. (2012). Claves de la política exterior de Colombia. Revista Latinoamérica, (54), 37-74. Disponible en: http://www. cialc.unam.mx/web_latino_final/ archivo_pdf/Lat54-37.pdf

Departamento Nacional de Planeación - DNP (2013). Pobreza monetaria y desigualdad del ingreso: Análisis de los resultados recientes. 2010-2012. Bogotá: DNP.

Flemes, D. (2012). La política exterior colombiana desde la perspectiva del realismo neoclásico. En: S. Jost (Ed), Colombia: una potencia en desarrollo. Escenarios y desafíos para su política exterior (pp.19-37). Bogotá: KAS.

Flemes, D. y Nolte, D. (2010). Introduction. Regional Leadership in the Global System: Ideas, Interests, and Strategies of Regional Powers. En: D. Flemes (Ed.), Regional Leadership in the Global System: Ideas, Interests, and Strategies of Regional Powers (pp. 1-14). Surrey: Ashgate Publishing Limited.

Fleury, S. (2012). Las reformas pendientes: desafíos para la gobernabilidad en Brasil. CIDOB d'afers internationals, 97-98.

Fraga, R. (2011). Conferencia del 5 de abril de 2011, Manhã. En: Fundação Alexandre de Gusmão. IX curso para Diplomatas Sul-Americanos. Brasilia: FUNAG. 
Godehardt, N. y Lembcke, O. (2010). Regionale Ordnungen in politischen Räumen. Ein Beitrag zur Theorie regionaler Ordnungen. Hamburg: German Institute of Global and Area Studies.

Herrera, B. (2012). La región andina y las incidencias geopolíticas en la inserción internacional del Estado colombiano. En: S. Jost (Ed), Colombia: una potencia en desarrollo. Escenarios y desafíos para su política exterior (pp.565-575). Bogotá: KAS.

Hooghe, L. y Marks, G. (2003). Unraveling the Central State, But How? Types of MultiLevel Governance. Vienna: Institute for Advanced Studies.

Hooghe, L. y Marks, G. (2004). Gobernanza estatocéntrica y multinivel. En: F. Morata (Ed), Gobernanza multinivel en la Unión Europea (pp. 51-85). Valencia: Tirant Lo Blanch.

Iniciativa para la Integración de la Infraestructura Regional Suramericana (2011). IIRSA 10 Años Después: Sus Logros y Desafíos. Disponible en: www.iirsa.org.

Iniciativa para la Integración de la Infraestructura Regional Suramericana (2012). Agenda de Proyectos Prioritarios. Informe de avance. Disponible en: www.iirsa.org.

Márquez, M. (2012). "Dilemas y perspectivas de la relación de Colombia con Venezuela en la era Santos”. En: Jost, S. (Ed). Colombia: una potencia en desarrollo. Escenarios y desafíos para su política exterior. Bogotá: KAS. pp. 576-598.

Nolte, D. (2006). Potencias regionales en la política internacional: conceptos y enfoques de análisis. Hamburgo: German Institute of Global and Area Studies.

Pastrana, E. (2011). Evolución y perspectivas de las relaciones entre Colombia y Brasil. Plataforma Democrática. Working Paper No. 14. Disponible en: http://www. plataformademocratica.org/Arquivos/Plataforma_Democratica_Working_Paper_14_2011_Espanhol.pdf

Pastrana, E. y Vera, D. (2012). "Rasgos de la política exterior brasileña en su proceso de ascenso como potencia regional y global”. En: Jost, S. (Ed). Colombia y Brasil: ¿socios estratégicos en la construcción de Suramérica? Bogotá: KAS, pp. 135-184

Pastrana, E. y Vera, D. (2012a). En: Jost, S. (Ed). Colombia y Brasil: ¿̇socios estratégicos en la construcción de Suramérica? Bogotá: KAS, pp. 135-184.

Salama, P. (2010). Brasil, el legado económico de Lula: éxitos y límites. Revista Ciclos, 19 (37-38), 3-18.

Solano, M. y Dangond, C. (2010). "El narcotráfico en Colombia como tema de la agenda internacional”. En: Jost, S., Pastrana, E., y Márquez, M. (Eds) Más allá de la seguridad democrática: agenda hacia nuevos horizontes. Bogotá: KAS-PUJ, pp. 431-450. 
Tavares, J. (2013). De la IIRSA al COSIPLAN: Desafíos de la Integración Física de América del Sur. En: Sorj, B. y Fausto, S. (Eds). Brasil y América Latina: ¿Qué Liderazgo es Posible? San Pablo: Plataforma Democrática, pp. 141-164

Tenjo, M (2009). Colombia: un modelo de desarrollo desde la IIRSA. Bogotá: Instituto Latinoamericano de Servicios Legales.

Tickner, A. (2007). Intervención por invitación. Claves de la política exterior colombiana y de sus debilidades principales. Colombia Internacional, 65, enero-junio. Bogotá: Universidad de los Andes, pp. 90-11.

UNASUR (2011). Infraestructura para la integración regional. Santiago de Chile: Naciones Unidas.

Telesur. (2012). Peña Nieto solicita apoyo en Brasil para luchar contra la pobreza en México. Disponible en: http://www.telesurtv.net/articulos/2012/o9/20/penanieto-solicita-apoyo-de-presidenta-brasilena-para-luchar-contra-la-pobrezaen-mexico-6515.html

Wills-Valderrama, S. (2011). Protección a la inversión extranjera en infraestructura por medio de Acuerdos Internacionales de Inversión: un nuevo reto para Colombia. International Law. Revista Colombiana de Derecho Internacional, 19, pp. 17-38. 
